\title{
Research Article \\ Roll Attitude Determination of Spin Projectile Based on GPS and Magnetoresistive Sensor
}

\author{
Dandan Yuan, Wenjun Yi, and Jun Guan \\ National Key Laboratory of Transient Physics, Nanjing University of Science and Technology, Nanjing 210094, China \\ Correspondence should be addressed to Dandan Yuan; tianhongjun@qq.com
}

Received 8 August 2017; Accepted 5 December 2017; Published 31 December 2017

Academic Editor: Hua Fan

Copyright (C) 2017 Dandan Yuan et al. This is an open access article distributed under the Creative Commons Attribution License, which permits unrestricted use, distribution, and reproduction in any medium, provided the original work is properly cited.

\begin{abstract}
Improvement in attack accuracy of the spin projectiles is a very significant objective, which increases the overall combat efficiency of projectiles. The accurate determination of the projectile roll attitude is the recent objective of the efficient guidance and control. The roll measurement system for the spin projectile is commonly based on the magnetoresistive sensor. It is well known that the magnetoresistive sensor produces a sinusoidally oscillating signal whose frequency slowly decays with time, besides the possibility of blind spot. On the other hand, absolute sensors such as GPS have fixed errors even though the update rates are generally low. To earn the benefit while eliminating weaknesses from both types of sensors, a mathematical model using filtering technique can be designed to integrate the magnetoresistive sensor and GPS measurements. In this paper, a mathematical model is developed to integrate the magnetoresistive sensor and GPS measurements in order to get an accurate prediction of projectile roll attitude in a real flight time. The proposed model is verified using numerical simulations, which illustrated that the accuracy of the roll attitude measurement is improved.
\end{abstract}

\section{Introduction}

Improvement in attack accuracy of the spin projectiles is a very important objective, which increases the overall combat efficiency of projectiles. The accurate determination of the spin projectile roll attitude has recently become the key technology for improving guidance accuracy and flight stability. Inertial Navigation System (INS) is commonly used to determine the position, speed, and attitude of projectile with a high update, which consists mainly of three gyroscopes and three accelerometers. The INS has commonly been used as a means of localization for projectiles [1-3]. The disadvantage in use of an INS, particularly when using low-cost sensors, is due to the accumulation of errors with time due to the dead-reckoning nature of the sensor. Furthermore, the inertial navigation can hardly satisfy the requirements of the projectile launching system such as high-g and high spin rate. The magnetoresistive sensor can be used to determine the roll attitude in effective way, especially in the high spin and high-g projectile launching environment. In addition, it can overcome the disadvantage of the accumulated errors over time. Moreover, it has advantages $[4,5]$ of passive sensing, high sensitivity, high-resolution measurement, low power, small size, and low cost. For example, HMC1043 has a linearity error of $0.1 \%$ Fs under best fit straight line $( \pm 1$ Gauss $)$ conditions, and the sensitivity is $1 \mathrm{mV} / \mathrm{V} /$ Gauss. So it has been widely and successfully used in attitude measurement [6, 7]. The GPS also is commonly used to determine the navigation data of projectile. It has advantages of high precision, all-weather capability, and continuous work, which can provide real-time information of position and velocity of projectile [8-10]. A method for synthesizing attitude data using single-antenna GPS velocity measurements was successfully demonstrated in this study [8]. The notion of pseudoattitude was introduced and was shown to provide adequate attitude data with no subjective or substantial objective differences as compared with the conventional attitude display.

In many practical applications, if only one kind of sensor is adopted for attitude measurement, the precision will be affected due to respective drawbacks and restrictions. Hence, some integrated sensors are emerged [11-14], such as GPS/ INS, GPS/MEMS-INS, integration of GPS and accelerometer, and integration of magnetoresistive sensor and gyroscope. Attitude determination systems that use inexpensive sensors, 
TABLE 1: Table on direction cosine between ground coordinate system and body coordinate system.

\begin{tabular}{lccc}
\hline & $o x_{1}$ & $o y_{1}$ & $o z_{1}$ \\
\hline$o x$ & $\cos \theta \cos \psi$ & $-\sin \theta \cos \psi \cos \gamma+\sin \psi \sin \gamma$ & $\sin \theta \cos \psi \sin \gamma+\sin \psi \cos \gamma$ \\
$o y$ & $\sin \theta$ & $\cos \theta \cos \gamma$ & $-\cos \theta \sin \gamma$ \\
$o z$ & $-\cos \theta \sin \psi$ & $\sin \theta \sin \psi \cos \gamma+\cos \psi \sin \gamma$ & $-\sin \theta \sin \psi \sin \gamma+\cos \psi \cos \gamma$ \\
\hline
\end{tabular}

which are based on computationally efficient and robust algorithms, are indispensable for real-time vehicle navigation, guidance, and control applications $[15,16]$. The accelerometers in conjunction with the derivative of GPS velocity provided a measure of the gravitation field vector and the magnetometers measured the earth's magnetic field vector.

In this paper, a mathematical model is developed to integrate the magnetoresistive sensor and GPS measurements in order to get an accurate prediction of projectile roll attitude in a real flight time. Based on the velocity information provided by GPS, the trajectory inclination angle and deflection angle are determined. And then the roll attitude can be calculated by using the developed model which utilizes the magnetoresistive sensor output. In this study [17], a high-frequency measuring method for the projectile roll angle and angular rate, which is based on the assumptions of small angle-ofattack and single axis rotation, is established using the flight characteristics of high-spin projectile. The real-time systematic error of this combined measuring system is calculated by establishing the simulation model with pitch angle as a variable. In this work, the typical magnetoresistive sensor is used for roll attitude measurement, and in the event of blind spot, GPS can give aiding data, enabling the navigation system to go on until the magnetoresistive output can be reestablished. Regarding the geomagnetic field characteristics, it can be seen that the intensity of geomagnetic field and its direction are function of position. And based on the calculation of the International Geomagnetic Reference Field (IGRF), the total amounts of the Earth magnetic field in our country are basically equal in all places. And, as known, with the variation of $1^{\circ}$ in latitude or longitude, the ground distance varies by approximately $110 \mathrm{~km}$. For the proposed projectile, which is used in this paper for roll attitude determination, the range is basically within $100 \mathrm{~km}$. Therefore, the magnitude and direction of local magnetic field are almost invariable with the range. The proposed model is verified using numerical simulations, which illustrated that the accuracy of the roll attitude measurement is improved.

\section{Principle on Measurement of Projectile Roll Attitude with GPS and Magnetoresistive Sensor}

Three attitude angles (Euler angles) of projectile describe the relation between body coordinate system $o-x_{1} y_{1} z_{1}$ and ground coordinate system $o-x y z . \theta, \psi$, and $\gamma$ refer to pitch angle, yaw angle, and projectile spinning angle, respectively. Transformation matrix between these two frames is noted as $A_{01}$, and corresponding transformation relation is listed in Table 1 .
Magnetoresistive sensor was embedded on the projectile; the direction of its sensitive axis should be consistent with direction of three axes in body coordinate system, which is used to measure the projection of the Earth magnetic field. The three components of geomagnetic field intensity, $\mathbf{B}$, in geographical coordinate system o-NED (North East Down) are denoted as $B_{N}, B_{E}$, and $B_{D}$, respectively. $B_{x}, B_{y}$, and $B_{z}$ refer to the projection of geomagnetic vector on the three axes of ground coordinate system. Angle $\phi$ is used to describe the angle between $o x$-axis of ground coordinate system and $o N$ axis of geographical coordinate system. $B_{x_{1}}, B_{y_{1}}$, and $B_{z_{1}}$ refer to components of geomagnetic vector in body coordinate system. Using geomagnetic information, the formula can be derived from coordinate systems transformation as follows:

$$
\begin{aligned}
{\left[\begin{array}{c}
B_{N} \\
B_{E} \\
B_{D}
\end{array}\right]=} & {\left[\begin{array}{ccc}
\cos (\phi) & 0 & -\sin (\phi) \\
\sin (\phi) & 0 & \cos (\phi) \\
0 & -1 & 0
\end{array}\right]\left[\begin{array}{c}
B_{x} \\
B_{y} \\
B_{z}
\end{array}\right] } \\
= & {\left[\begin{array}{ccc}
\cos (\phi) & 0 & -\sin (\phi) \\
\sin (\phi) & 0 & \cos (\phi) \\
0 & -1 & 0
\end{array}\right] A_{01}\left[\begin{array}{l}
B_{x_{1}} \\
B_{y_{1}} \\
B_{z_{1}}
\end{array}\right] . }
\end{aligned}
$$

Generally, angle $\phi$ is the fixed and known parameter. In this work, the $o x$-axis of ground coordinate system is coincided with $o N$-axis of geographic coordinate system, so, namely, $\phi=0$.

Thus, formula (1) can be simplified and transformed into

$$
\begin{aligned}
B_{D}+\sin \theta B_{x_{1}} & =\cos \theta\left(\sin \gamma B_{z_{1}}-\cos \gamma B_{y_{1}}\right), \\
B_{N} \sin \psi+B_{E} \cos \psi & =\sin \gamma B_{y_{1}}+\cos \gamma B_{z_{1}} .
\end{aligned}
$$

Hence, calculation formula of projectile spinning angle can be obtained from the above formula:

$$
\gamma=\arctan \left(\frac{q B_{z_{1}}+p B_{y_{1}}}{p B_{z_{1}}-q B_{y_{1}}}\right),
$$

where

$$
\begin{gathered}
q=\frac{\left(B_{D}+\sin \theta B_{x_{1}}\right)}{\cos \theta}, \\
p=B_{N} \sin \psi+B_{E} \cos \psi .
\end{gathered}
$$

It can be derived from analysis of formula (3) that projectile spinning angle can be calculated conveniently if the pitch angle and yaw angle are replaced by trajectory inclination angle and deflection angle, respectively. Although replacement of attitude angles can lead to certain error, the feasibility 
of such replacement has been verified. Usually, attack angle is very small during the projectile flight aiming to ensure flight stability, while it also assures the effectiveness of the replacement, and then the error caused by the replacement under flight of small attack angle can be neglected.

Using the components $v_{N x}, v_{E y}$, and $v_{D z}$ of projectile velocity in the NED coordinate system, which is provided by GPS, trajectory inclination angle and trajectory deflection angle can be calculated. From the definition of trajectory inclination angle, which describes the angle between horizontal plane of ground coordinate system and velocity vector, the formula of trajectory inclination angle can be written as

$$
\theta_{v}=\arctan \left(-\frac{v_{D z}}{\sqrt{v_{N x}^{2}+v_{E y}^{2}}}\right) .
$$

From the definition of trajectory deflection angle, which describes the relation between the projection of velocity vector on horizontal plane of ground coordinate system and the $o x$-axis, the formula of trajectory deflection angle can be written as

$$
\psi_{v}=\arctan \left(\frac{v_{E y}}{v_{N x}}\right) .
$$

In above parts, the detailed approach to measure and calculate projectile spinning angle utilizing the combination of GPS and magnetoresistive sensor outputs is described. However, in the projectile flight, outputs of magnetoresistive sensor installed on $y_{1}$-axis and $z_{1}$-axis of projectile depend on the position relation between geomagnetic field vector and the projectile body axis. In flight, when the direction of geomagnetic field intensity is lying on the firing plane, the component of geomagnetic field intensity on lateral axis of projectile will be zero. So the output of sensitive axis of magnetoresistive sensor on lateral axis is noise only. In this situation, calculation of projectile spinning angle is somewhat limited. Similarly, at certain moment in flight, if the projectile axis is paralleled or nearly paralleled to geomagnetic field vector, the projection of geomagnetic field intensity on longitudinal plane of carrier is zero, and calculation result of projectile spinning angle is unreliable. Therefore, in measurement of projectile spinning angle, possible blind spot of magnetic measurement will exist. And then the projectile spinning angle will be inaccurate. In order to solve the problem of blind spot of magnetic measurement, roll angle relative to velocity vector axis is calculated according to GPS output, which is used as a compensation to the inaccurate projectile spinning angle. Note that the roll angle relative to velocity vector axis is referred to as the projectile pseudoroll angle. Moreover, relevant mathematical model is established and the feasibility of the compensation is demonstrated.

\section{Solution of Projectile Pseudoroll Angle}

For the purposes of coordinates description, trajectory-fixed coordinate system $o-x_{2} y_{2} z_{2}$ and velocity coordinate system $o-x_{3} y_{3} z_{3}$ are defined. The trajectory-fixed coordinate origin,

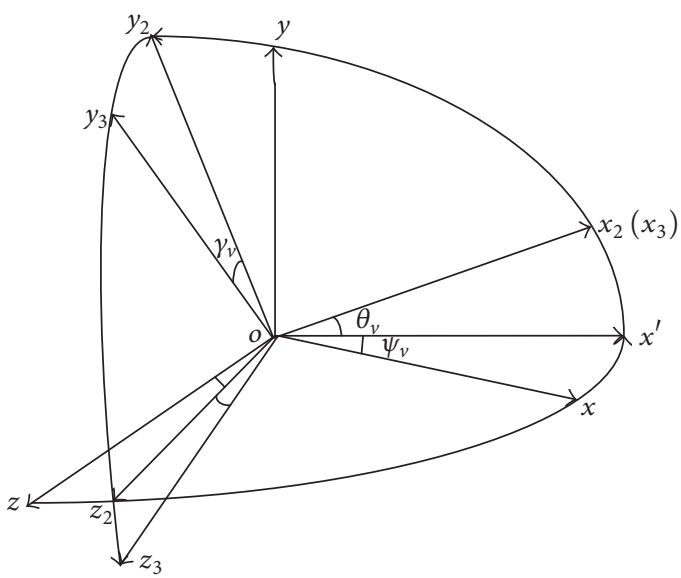

FIGURE 1: Relations between three coordinate systems.

$o$, is located in the projectile's center of mass. The $o x_{2}$-axis is coincided with velocity vector. The $o y_{2}$-axis is perpendicular to the $o x_{2}$-axis and is located in the vertical plane, while the $o z_{2}$-axis is determined by completing the right-hand rule. The velocity coordinate origin, $o$, is also located in the projectile's center of mass, and the $o x_{3}$-axis is also coincided with velocity vector. The $o y_{3}$-axis is perpendicular to the $o x_{3}$ axis and is located in longitudinal symmetry plane of the projectile, while the $o z_{3}$-axis is determined by completing the right-hand rule. According to their definitions, the relation between ground coordinate system and trajectoryfixed coordinate system is derived by using two angles, while the relation between trajectory-fixed coordinate system and velocity coordinate system can be derived by using one angle. Mutual relations between the three coordinates can be seen clearly from Figure 1.

3.1. Calculation of Projectile Pseudoroll Angle. By using i, j, and $\mathbf{k}$ to represent unit vectors along three axes of NED coordinate system, the velocity components and acceleration components of projectile in the NED coordinate system can be shown as

$$
\begin{aligned}
& \mathbf{v}=v_{N x} \mathbf{i}+v_{E y} \mathbf{j}+v_{D z} \mathbf{k}, \\
& \mathbf{a}=a_{N x} \mathbf{i}+a_{E y} \mathbf{j}+a_{D z} \mathbf{k} .
\end{aligned}
$$

Acceleration can be estimated by the velocity $\left(v_{N x}\right.$, $v_{E y}, v_{D z}$ ) from GPS output, and the calculation of longitudinal acceleration is taken as instance to make explanation. The longitudinal velocities at the moments $k$ and $k+1$ are denoted as $v_{N x}(k)$ and $v_{N x}(k+1)$, respectively. So the longitudinal acceleration at moment $k+1$ can be calculated as follows:

$$
a_{N x}(k+1)=\frac{\left(v_{N x}(k+1)-v_{N x}(k)\right)}{\left(t_{k+1}-t_{k}\right)} .
$$

Thus, the steps to calculate projectile pseudoroll angle are listed below.

(1) Resolve of Acceleration in $\mathrm{y}_{3} z_{3}$ Plane of Velocity Coordinate System. The acceleration vector a is resolved into tangential acceleration $\mathbf{a}^{t}$ and normal acceleration $\mathbf{a}^{n}$ along velocity 
vector $\mathbf{v}$, while the angle between acceleration vector and velocity vector is marked as $\phi_{a}$. The gravitational acceleration vector $\mathbf{g}$ is resolved into tangential component $\mathbf{g}^{t}$ and normal component $\mathbf{g}^{n}$ along velocity vector $\mathbf{v}$, while the angle between gravitational acceleration vector and velocity vector is marked as $\phi_{g}$. Obviously, both the normal acceleration $\mathbf{a}^{n}$ and the normal gravitational acceleration $\mathbf{g}^{n}$ are in longitudinal plane $o y_{3} z_{3}$, which is vertical to the velocity. According to the principle of physical dynamic balance, we can see that $\mathbf{a}^{n}$ is a total tangential acceleration including vector $\mathbf{g}^{n}$ and another acceleration vector $\mathbf{l}$, so vector $\mathbf{l}=\mathbf{a}^{n}-\mathbf{g}^{n}$. Calculations involved are listed as follows:

$$
\begin{aligned}
\phi_{a} & =\arccos \left(\frac{(\mathbf{a} \cdot \mathbf{v})}{|\mathbf{a}||\mathbf{v}|}\right), \\
\mathbf{a}^{t} & =|\mathbf{a}| \cos \left(\phi_{a}\right) \frac{\mathbf{v}}{|\mathbf{v}|} \\
& =\left(\frac{|\mathbf{a}| \cos \left(\phi_{a}\right)}{|\mathbf{v}|}\right)\left(v_{N x} \mathbf{i}+v_{E y} \mathbf{j}+v_{D z} \mathbf{k}\right), \\
\mathbf{a}^{n} & =\mathbf{a}-\mathbf{a}^{t} \\
\phi_{g} & =\arccos \left(\frac{(\mathbf{g} \cdot \mathbf{v})}{|\mathbf{g}||\mathbf{v}|}\right), \\
\mathbf{g}^{t} & =|\mathbf{g}| \cos \left(\phi_{g}\right) \frac{\mathbf{v}}{|\mathbf{v}|} \\
& =\left(\frac{|\mathbf{g}| \cos \left(\phi_{g}\right)}{|\mathbf{v}|}\right)\left(v_{N x} \mathbf{i}+v_{E y} \mathbf{j}+v_{D z} \mathbf{k}\right), \\
\mathbf{g}^{n} & =\mathbf{g}-\mathbf{g}^{t} .
\end{aligned}
$$

(2) Direction of Resultant Force in oy $z_{3}$ Plane of Velocity Coordinate System. It is known from exterior ballistics theory that, because of the existence of angle of attack, the direction of projectile velocity and the projectile axis are not coincident. Therefore, total aerodynamic force includes not only drag aligned with the opposite direction of the velocity but also lift which is vertical to velocity and located in $o y_{3} z_{3}$ plane of velocity coordinate system. According to Newton's second law of motion, we can see that the direction of lift force is consistent with the direction of acceleration vector $\mathbf{l}$. The lift force $f_{y_{3} z_{3}}$ in $o y_{3} z_{3}$ plane can be decomposed into force $f_{y_{3}}$ and force $f_{z_{3}}$ along the $o y_{3}$-axis and the $o z_{3}$-axis, respectively, shown as Figure 2.

The angle $\phi_{\gamma}$ between the lift $f_{y_{3} z_{3}}$ and $o y_{3}$-axis can be calculated as

$$
\phi_{\gamma}= \begin{cases}\arctan \left(\frac{f_{z_{3}}}{f_{y_{3}}}\right) & f_{y_{3}}>0, f_{z_{3}} \geq 0 \\ 2 \pi+\arctan \left(\frac{f_{z_{3}}}{f_{y_{3}}}\right) & f_{y_{3}}>0, f_{z_{3}}<0 \\ \pi+\arctan \left(\frac{f_{z_{3}}}{f_{y_{3}}}\right) & f_{y_{3}}<0 \\ \frac{\pi}{2}, & f_{y_{3}}=0, f_{z_{3}} \geq 0 \\ \frac{3 \pi}{2} & f_{y_{3}}=0, f_{z_{3}}<0 .\end{cases}
$$

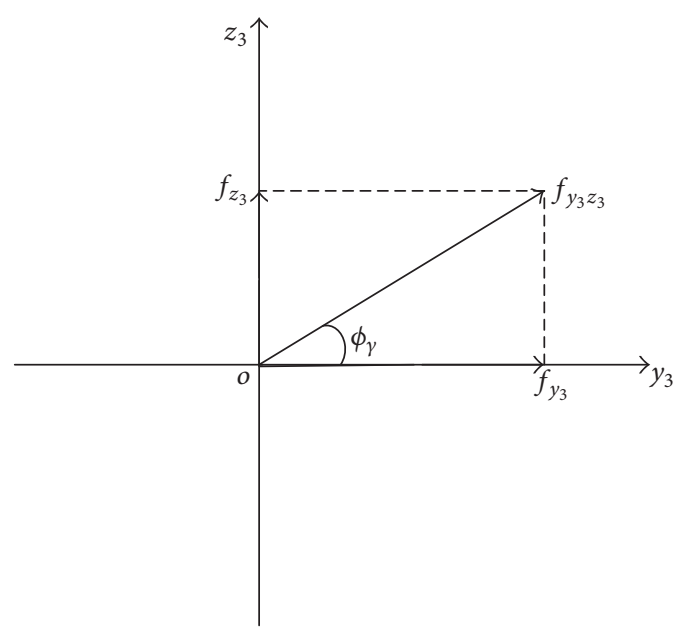

FIGURE 2: Distribution of lift in longitudinal plane $o y_{3} z_{3}$.

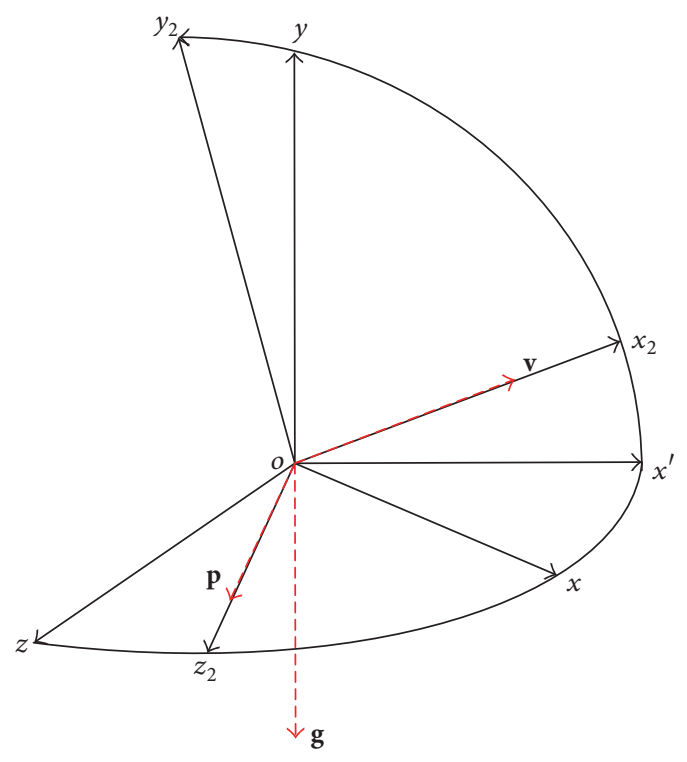

FIGURE 3: Direction of horizontal parameters.

(3) Construction of Horizontal Parameter. Horizontal parameter vector $\mathbf{p}$ can be constructed according to the relation between gravitational acceleration vector and velocity vector, represented as $\mathbf{p}=\mathbf{g} \times \mathbf{v}$. It is located in horizontal reference plane, and its direction is aligned with the direction of $o z_{2}$ axis in trajectory-fixed coordinate system, which is shown in Figure 3 as red-dotted line.

(4) Calculation of the Angle between Vector $\mathbf{l}$ and Vertical Plane. The angle between acceleration vector $\mathbf{l}$ and the constructed horizontal parameter vector $\mathbf{p}$ can be solved with the following formula:

$$
\phi=\arccos \left(\frac{(\mathbf{l} \cdot \mathbf{p})}{(|\mathbf{l}||\mathbf{p}|)}\right) .
$$




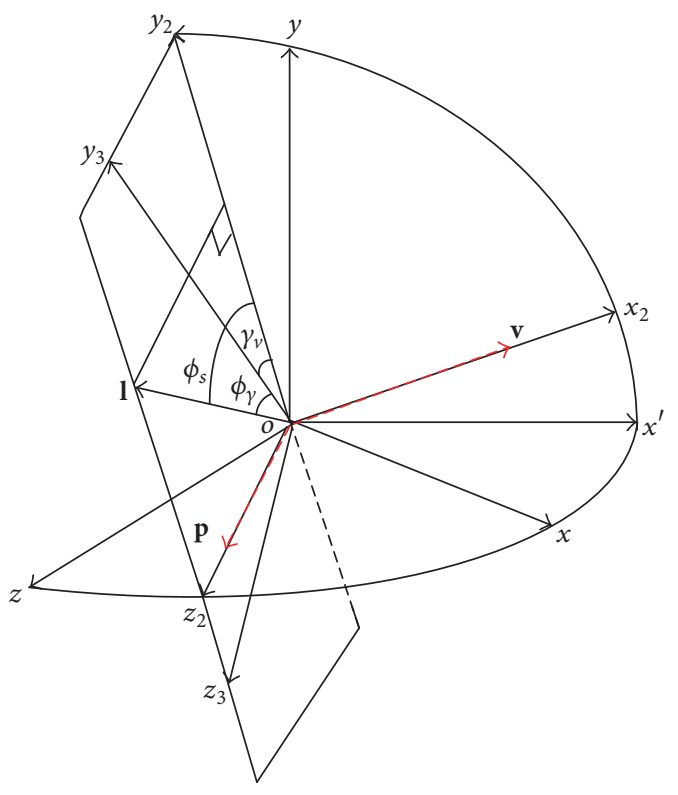

FIGURE 4: Relations among three angles.

The coordinate of vector $\mathbf{l}$ in ground coordinate system is denoted as $\mathbf{l}_{1}=\left(\begin{array}{lll}l_{x} & l_{y} & l_{z}\end{array}\right)$, and then

$$
\mathbf{l}_{1}=\left[\begin{array}{ccc}
1 & 0 & 0 \\
0 & 0 & -1 \\
0 & 1 & 0
\end{array}\right] \cdot \mathbf{l} .
$$

The angle $\phi_{s}$ between acceleration vector $\mathbf{l}$ and vertical plane is calculated as follows:

$$
\phi_{s}= \begin{cases}\phi-\frac{\pi}{2} & l_{y}>0, l_{z} \leq 0 \\ \frac{\pi}{2}-\phi & l_{y} \geq 0, l_{z}>0 \\ \frac{\pi}{2}+\phi & l_{y}<0 .\end{cases}
$$

(5) Calculation of Projectile Pseudoroll Angle. Using the relation among the three angles $\phi_{\gamma}, \phi_{s}$, and $\gamma_{v}$ which is shown in Figure 4, the projectile pseudoroll angle can be solved as follows:

$$
\gamma_{v}=\phi_{s}-\phi_{r}
$$

3.2. Verifying Feasibility of Replacement. According to the exterior ballistics theory, the relation among ground, trajectory-fixed, velocity, and body coordinate systems is correlated by eight angles $\left(\theta, \psi, \gamma, \alpha, \beta, \theta_{v}, \psi_{v}, \gamma_{v}\right)$, and they are not independent completely [18]. It is known that the cosine angle between two unit vectors, which both pass through the origin of the reference system, is equivalent to the sum of the product of the cosine angles between each unit vector and the three respective axes of the reference system. Body coordinate system is taken as the reference system, and one unit vector is along the direction of $\mathrm{oz}_{3}$-axis of velocity coordinate system, while the other unit vector is along the direction of $o y$-axis of ground coordinate system. Then, the geometrical relationship equation can be established as follows:

$$
\sin \left(\gamma_{v}\right)=\frac{(\cos (\alpha) \sin (\beta) \sin (\theta)-\sin (\alpha) \sin (\beta) \cos (\gamma) \cos (\theta)+\cos (\beta) \sin (\gamma) \cos (\theta))}{\cos \left(\theta_{v}\right)},
$$

where $\alpha$ refers to angle of attack and $\beta$ refers to angle of side slip.

Based on the assumption of small angle of attack, it can be considered that $\alpha$ and $\beta$ are small; therefore there are approximate relations shown as follows:

$$
\begin{aligned}
& \sin (\alpha) \approx \alpha, \\
& \cos (\alpha) \approx 1,
\end{aligned}
$$

$$
\begin{aligned}
& \sin (\beta) \approx \beta, \\
& \cos (\beta) \approx 1 .
\end{aligned}
$$

Using the projectile axis and velocity coordinate system, the following relations can be derived:

$$
\begin{aligned}
& \theta=\alpha+\theta_{v}, \\
& \psi=\beta+\psi_{v} .
\end{aligned}
$$

Then (15) can be rewritten as

$$
\sin \left(\gamma_{v}\right) \approx \frac{\left(\beta \sin \left(\theta_{v}\right)+\alpha \beta \cos \left(\theta_{v}\right)-\alpha \beta \cos (\gamma) \cos \left(\theta_{v}\right)+\alpha^{2} \beta \cos (\gamma) \sin \left(\theta_{v}\right)+\sin (\gamma) \cos \left(\theta_{v}\right)-\alpha \sin (\gamma) \sin \left(\theta_{v}\right)\right)}{\cos \left(\theta_{v}\right)}
$$


Generally, the range of trajectory inclination angle is $(-\pi / 2, \pi / 2)$. Therefore,

$$
\begin{gathered}
-1<\sin \left(\theta_{v}\right)<1, \\
0<\cos \left(\theta_{v}\right)<1 .
\end{gathered}
$$

Take simultaneously the limit of both sides of (18), which are shown as follows:

$$
\begin{aligned}
& \lim _{\substack{\alpha \rightarrow 0 \\
\beta \rightarrow 0}} \sin \left(\gamma_{v}\right) \cos \left(\theta_{v}\right)=\sin \left(\gamma_{v}\right) \cos \left(\theta_{v}\right), \\
& \lim _{\substack{\alpha \rightarrow 0 \\
\beta \rightarrow 0}}\left(\beta \sin \left(\theta_{v}\right)+\alpha \beta \cos \left(\theta_{v}\right)-\alpha \beta \cos (\gamma) \cos \left(\theta_{v}\right)\right. \\
& +\alpha^{2} \beta \cos (\gamma) \sin \left(\theta_{v}\right)+\sin (\gamma) \cos \left(\theta_{v}\right) \\
& \left.-\alpha \sin (\gamma) \sin \left(\theta_{v}\right)\right)=\lim _{\substack{\alpha \rightarrow 0 \\
\beta \rightarrow 0}} \beta \sin \left(\theta_{v}\right)+\lim _{\substack{\alpha \rightarrow 0 \\
\beta \rightarrow 0}} \alpha \beta \\
& \cdot \cos \left(\theta_{v}\right)-\lim _{\substack{\alpha \rightarrow 0 \\
\beta \rightarrow 0}} \alpha \beta \cos (\gamma) \cos \left(\theta_{v}\right)+\lim _{\substack{\alpha \rightarrow 0 \\
\beta \rightarrow 0}} \alpha^{2} \beta \\
& \cdot \cos (\gamma) \sin \left(\theta_{v}\right)+\lim _{\substack{\alpha \rightarrow 0 \\
\beta \rightarrow 0}} \sin (\gamma) \cos \left(\theta_{v}\right)-\lim _{\substack{\alpha \rightarrow 0 \\
\beta \rightarrow 0}} \alpha \\
& \cdot \sin (\gamma) \sin \left(\theta_{v}\right) \\
& =0+\lim _{\substack{\alpha \rightarrow 0 \\
\beta \rightarrow 0}} \sin (\gamma) \cos \left(\theta_{v}\right) \\
& =\sin (\gamma) \cos \left(\theta_{v}\right) \\
& \Longrightarrow \sin \left(\gamma_{v}\right) \cos \left(\theta_{v}\right) \approx \sin (\gamma) \cos \left(\theta_{v}\right) \text {, } \\
& \text { namely } \sin \left(\gamma_{v}\right) \approx \sin (\gamma) \\
& \Longrightarrow \gamma_{V} \approx \gamma+2 k \pi \\
& \text { or } \gamma_{V} \approx(2 k+1) \pi-\gamma \text {, }
\end{aligned}
$$

where $k$ is integer number.

From the definition of ground, trajectory-fixed, velocity, and body coordinate systems, it can be known that both projectile pseudoroll angle and projectile spinning angle are within the same range at the same moment, so multiplevalued situations are excluded. Therefore,

$$
\gamma_{v} \approx \gamma
$$

\section{Simulation Verification}

Towards the measurement of projectile spinning angle using GPS and magnetoresistive sensor and the calculated method of projectile pseudoroll angle, a numerical simulation is used to verify their effectiveness and feasibility. Trajectory simulation is developed based on a full six degree-of-freedom rigid-projectile model. This simulation has been performed to generate the trajectory parameters as a reference in the following flight conditions: muzzle velocity of the projectile $v_{0}=750 \mathrm{~m} / \mathrm{s}$, total mass $m=43.25 \mathrm{~kg}$, and caliber $d=$ $130 \mathrm{~mm}$. World Magnetic Model (WMM) is issued every 5 years, and the latest one will expire by the end of 2019. Nanjing is located at 32.028-degree north latitude and 118.854-degree east longitude, with a height of 24.03 meters. According to WMM and geographic location of Nanjing, geomagnetic factors of Nanjing can be obtained by calculation; that is, $B_{N}=32827.3, B_{E}=-3153.2$, and $B_{D}=37268.2$. The velocity output of GPS and the three-axial output of magnetoresistive sensor can be simulated according to theoretical trajectory parameters. Finally, projectile spinning angle is calculated according to the algorithm presented in this work and then compared with roll angle generated by exterior ballistics. The simulation results are shown in Figures 5-9.

Figure 5 compares the calculation result of projectile spinning angle with its theoretical value. The left one shows the comparison about projectile spinning angle curve in whole flying process, while in the right one, the comparison curve of whole process is enlarged locally to see the coincidence more clearly. It can be seen from Figure 5 that, without taking blind spot of magnetic measurement into consideration, projectile spinning angle can be calculated effectively by the method with the combination of GPS and magnetoresistive sensor. The curve of error between calculation result of projectile spinning angle and its theoretical value is shown in Figure 6. The magnitude of the error is used to describe the precision of projectile roll attitude calculated with GPS and magneticresistive sensor combination, and simulation results indicate that high precision can be obtained by this method.

The projectile pseudoroll angle is calculated according to the proposed method using GPS output and compared with its theoretical value. The error of comparison is shown in Figure 7; it can be seen from the error curve that the method proposed in this work is effective and feasible to solve the projectile pseudoroll angle.

Figure 8 compares the results of projectile roll attitude calculation. The full line depicts projectile spinning angle calculated with the combination method, while the dotted line reflects the calculation result of the projectile pseudoroll angle based on GPS measurement only. Besides, the error curves of the two methods are shown in Figure 9. It can be seen that both of the errors are within range of $0.5^{\circ}$, and the calculation error is smaller with the GPS measurement only. Therefore, if blind spot of magnetic measurement appears in flight, the result of projectile attitude determination based on the combination of GPS and magnetoresistive sensor would be inaccurate. And the method based on GPS measurement only can be adopted to calculate the projectile pseudoroll angle which can efficiently substitute the invalid projectile spinning angle solved by the method with GPS and magnetoresistive sensor combination.

At last, we talk about the effect on the accuracy of the model in the geomagnetic anomaly environment and the electromagnetic environment on battleship. Magnetic anomaly is a local variation in the Earth's magnetic field resulting from variations in the magnetism of the rocks. From geomagnetic theory, in general, the interference of variations in the solar activity on the Earth's magnetic field is only tens of nanotesla. When the intensity of geomagnetic anomaly 


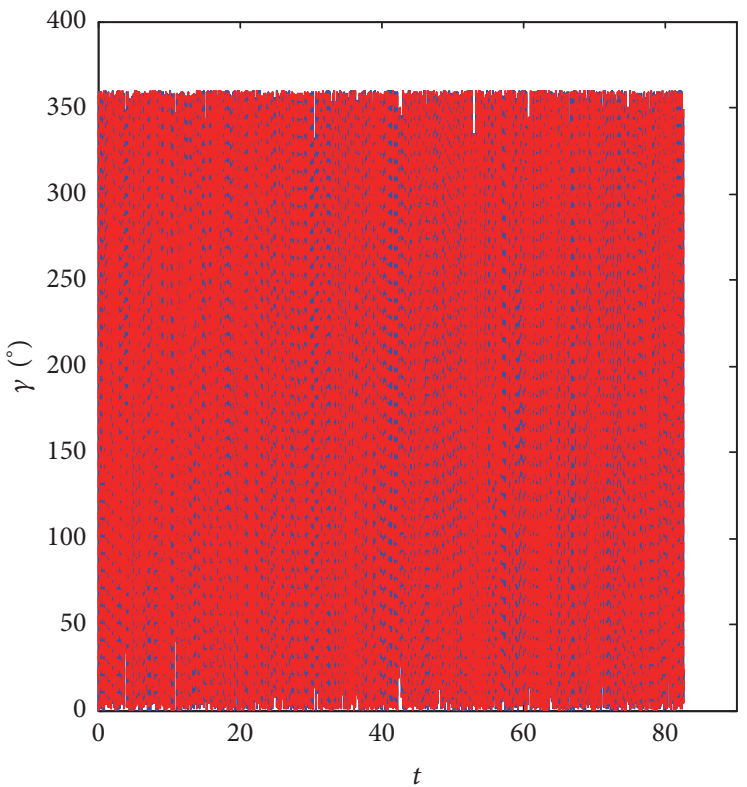

Solution value of roll angle

- - - Actual value of roll angle

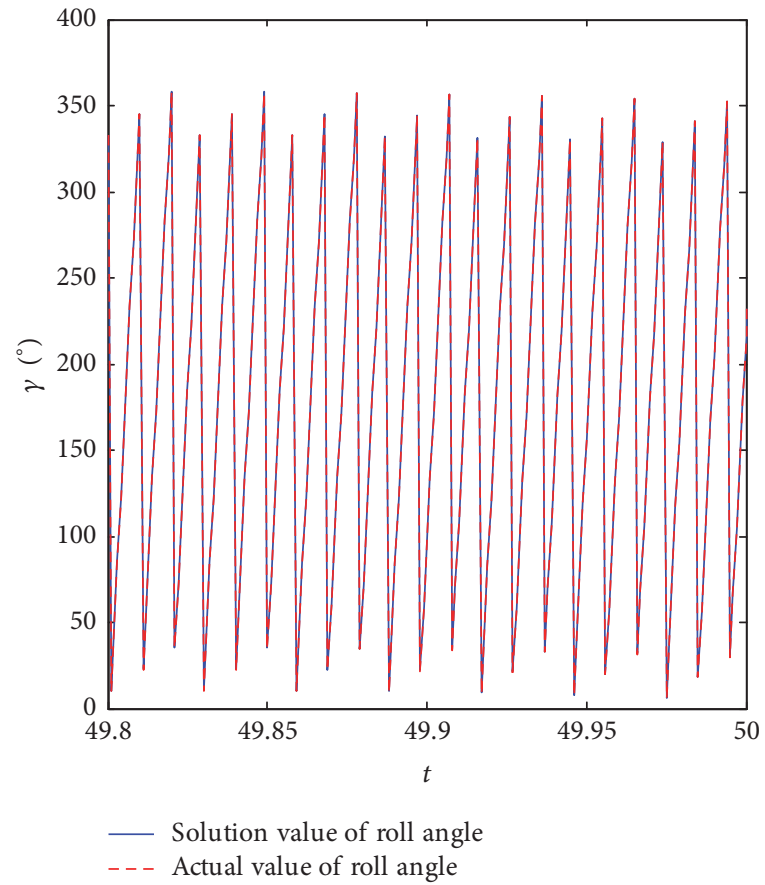

FIGURE 5: Calculation result and actual value of projectile roll attitude.

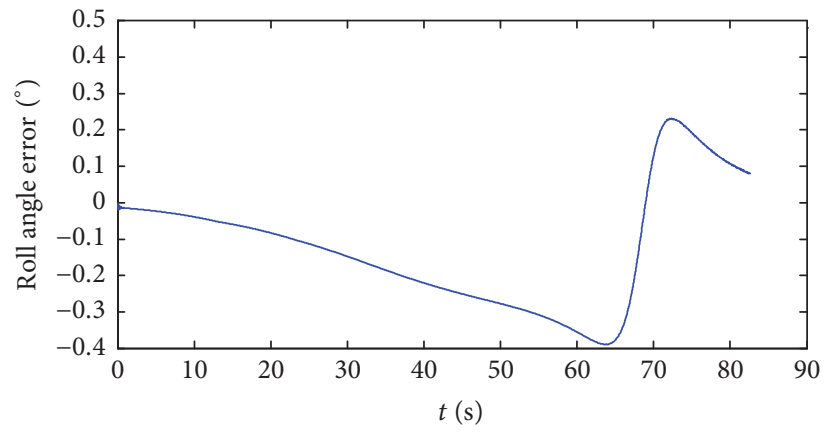

FIGURE 6: Calculation error of the combination method.

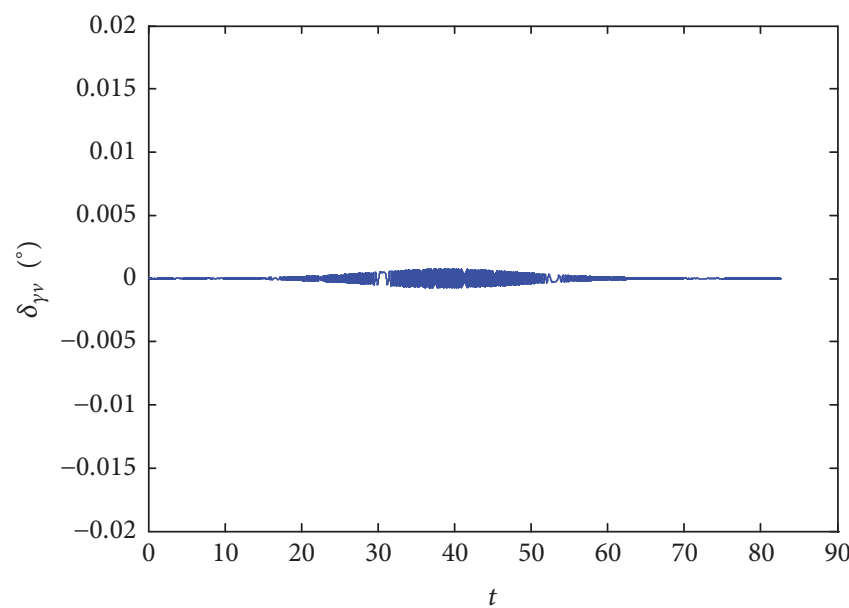

FIGURE 7: Error between calculation and actual value of roll angle relative to velocity. 


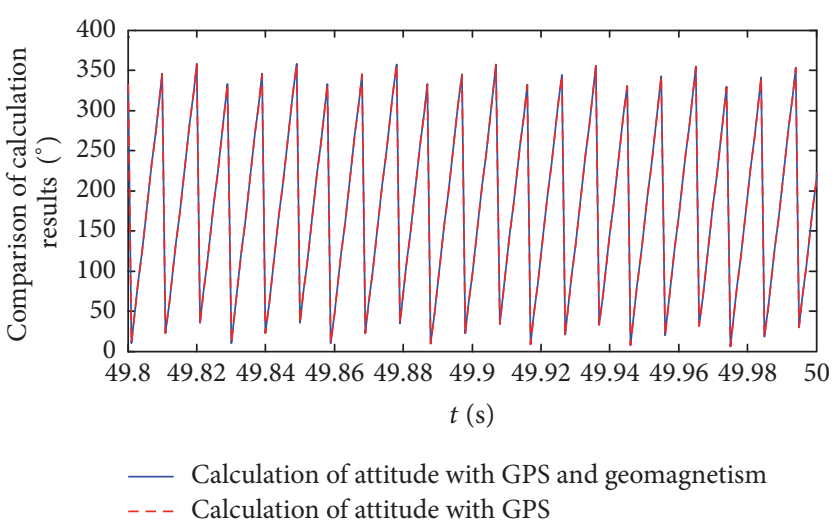

FIGURE 8: Comparison of calculation value with two methods (partial).

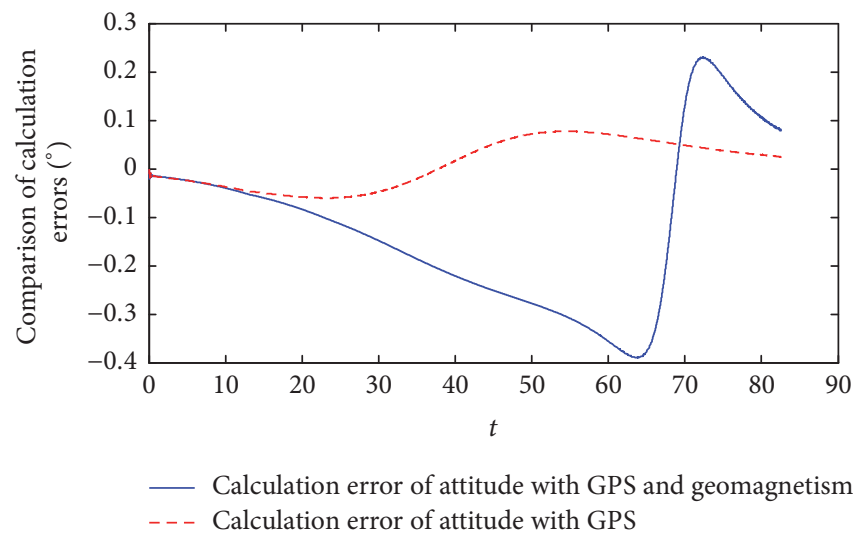

FIGURE 9: Comparison of attitude error.

is 100 nanotesla, the effect on the accuracy of the attitude measurement method is about 1.2 degrees. The simulation experiment regarding the electromagnetic environment on battleship has been carried out to test the working performance of the composite attitude measurement device. It shows that the composite attitude measurement device can work properly without the influence on measurement accuracy.

\section{Conclusion}

In this paper, a mathematical model is developed to integrate the magnetoresistive sensor and GPS measurements in order to get an accurate prediction of projectile spinning angle in a real flight time. It can be seen that the error between numerical simulation value and theoretical value of roll angle is within range of $1^{\circ}$, and it is a satisfactory precise solution that meets the requirement. In the event of blind spot of magnetic measurement, the three-axial output of magnetoresistive sensor will be invalid, and then the roll attitude calculation will have too large error and sequentially will affect the guidance and control precision. In this time, utilizing the aiding data given by the GPS, another new calculation model is built to solve the projectile pseudoroll angle, which enables the navigation system to go on until the magnetoresistive output can be reestablished. This new method can overcome the blind area drawback of magnetoresistive sensor effectively and improve precision in measurement of projectile roll attitude. In addition, the feasibility to replace projectile spinning angle with projectile pseudoroll angle is verified.

\section{Conflicts of Interest}

The authors declare that there are no conflicts of interest regarding the publication of this paper.

\section{Acknowledgments}

This work is supported by the National Natural Science Fund (no. 11472136).

\section{References}

[1] J.-B. Lacambre, M. Narozny, and J.-M. Louge, "Limitations of the unscented Kalman filter for the attitude determination on an inertial navigation system," in Proceedings of the 2013 IEEE Digital Signal Processing and Signal Processing Education Meeting, DSP/SPE 2013, pp. 187-192, USA, August 2013.

[2] P. Henkel and C. Günther, "Attitude determination with lowcost GPS/INS," in Proceedings of the 26th International Technical Meeting of the Satellite Division of the Institute of Navigation, pp. 2015-2023, Nashville, TN, USA, September 2013.

[3] S. Han and J. Wang, "Integrated GPS/INS navigation system with dual-rate Kalman Filter," GPS Solutions, vol. 16, no. 3, pp. 389-404, 2012. 
[4] A. Edelstein, "Advances in magnetometry," Journal of Physics: Condensed Matter, vol. 19, no. 16, Article ID 165217, 2007.

[5] P. Ripka and M. Janošek, "Advances in magnetic field sensors," IEEE Sensors Journal, vol. 10, no. 6, pp. 1108-1116, 2010.

[6] M. Díaz-Michelena, "Small magnetic sensors for space applications," Sensors, vol. 9, no. 4, pp. 2271-2288, 2009.

[7] S. Changey, D. Beauvois, and V. Fleck, "A Mixed ExtendedUnscented Filter for attitude estimation with magnetometer sensor," in Proceedings of the American Control Conference, pp. 2892-2897, June 2006.

[8] R. P. Kornfeld, H. R. John, and J. J. Deyst, "Single-antenna GPSbased aircraft attitude determination," Navigation, vol. 45, no. 1 , pp. 51-60, 1998.

[9] I. Y. Bar-Itzhack, P. Y. Montgomery, and J. C. Garrick, "Algorithms for attitude determination using GPS," in Proceedings of the Guidance, Navigation, and Control Conference, 1997, pp. 841851, August 1997.

[10] G. Falco, G. A. Einicke, J. T. Malos, and F. Dovis, "Performance analysis of constrained loosely coupled GPS/INS integration solutions," Sensors, vol. 12, no. 11, pp. 15983-16007, 2012.

[11] G. Schmidt, "INS/GPS Integration Architectures," RTO Lecture Series RTO-EN-SET-116, 2010.

[12] O. S. Salychev, V. V. Voronov, and M. E. Cannon, "Attitude determination with GPS-aided inertial navigation systems," in Proceedings of the Iain World Congress \& Annual Meeting of the Institute of Navigation, vol. 18, pp. 1-17, 2000.

[13] S. Changey, E. Pecheur, and T. Brunner, "Attitude Estimation of a projectile using Magnetometers and Accelerometers: Experimental Validation," in Proceedings of the 2014 IEEE/ION Position, Location and Navigation Symposium, PLANS 2014, pp. 1168-1173, USA, May 2014.

[14] H. E. Soken and C. Hajiyev, "In flight magnetometer calibration via unscented Kalman filter," in Proceedings of the 5th International Conference on Recent Advances in Space Technologies (RAST '11), pp. 885-890, June 2011.

[15] D. Gebre-Egziabher, G. H. Klkaim, J. Powell, and B. W. Parkinson, "A Gyro-free quaternion-based attitude determination system suitable for implementation using low cost sensors," in Proceedings of the IEEE Position, Location, and Navigation Symposium, pp. 185-192, March 2000.

[16] D. Gebre-Egziabher and G. H. Elkaim, "MAV attitude determination by vector matching," IEEE Transactions on Aerospace and Electronic Systems, vol. 44, no. 3, pp. 1012-1028, 2008.

[17] P. Cao, J. Y. Yu, and X. M. Wang, "High-frequency Measurement and Calculation study of Systematic Error of High-rolling Projectile Roll Angle Based on Combination of MR/GNSS," Journal of China Ordnance, vol. 35, no. 6, pp. 795-800, 2014.

[18] X. F. Qian, R. X. Lin, and Y. N. Zhao, Flight Mechanics of Guided Missile, Beijing Institute of Technology Press, Beijing, China. 


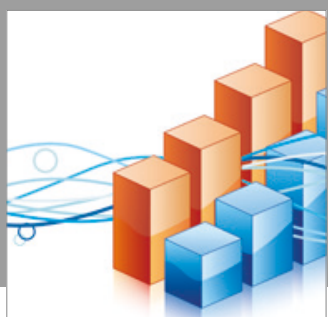

Advances in

Operations Research

vatersals

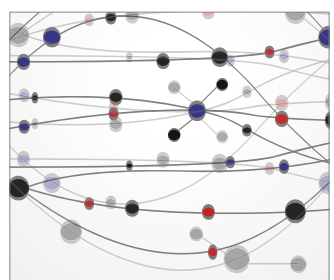

\section{The Scientific} World Journal
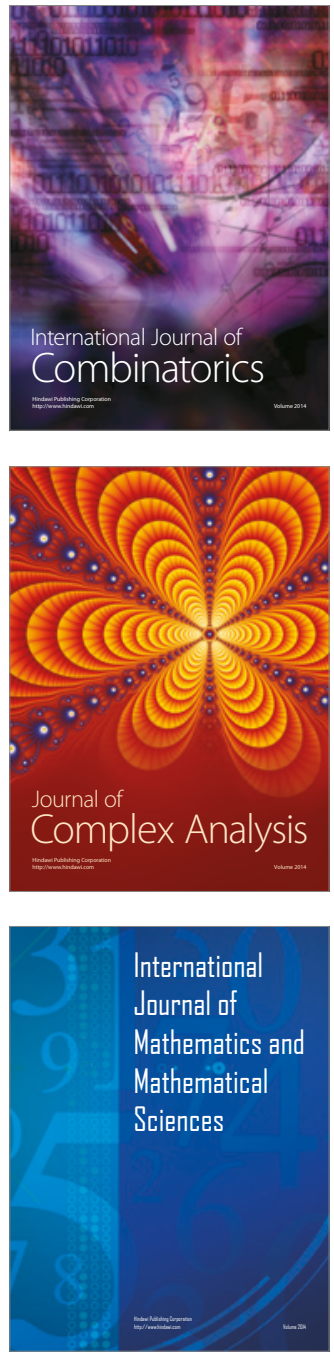
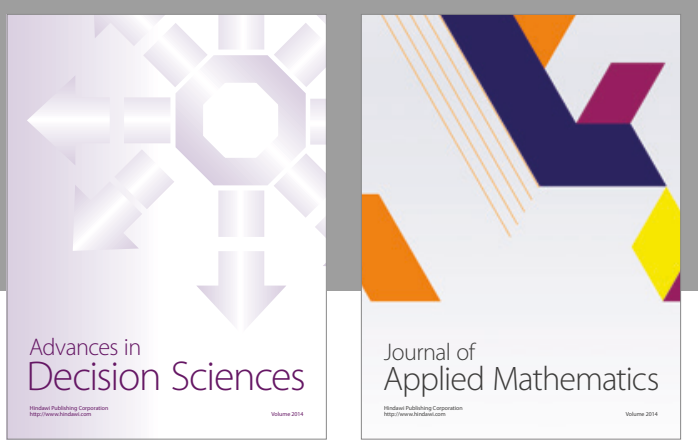

Algebra

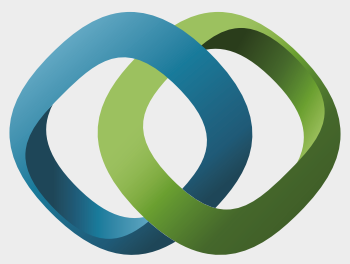

\section{Hindawi}

Submit your manuscripts at

https://www.hindawi.com
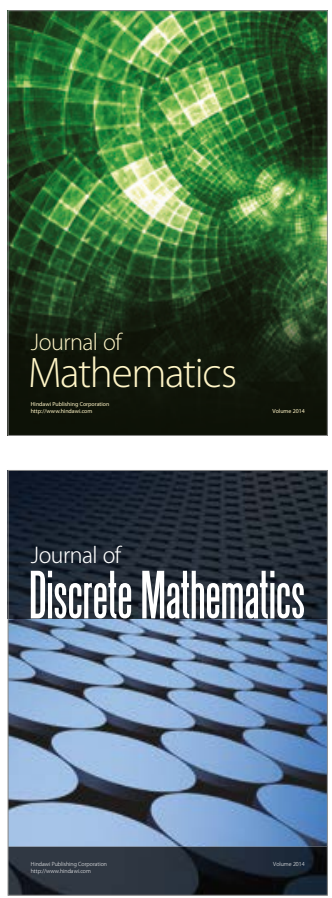

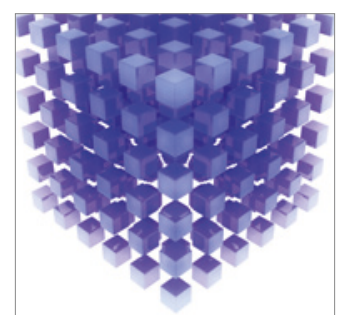

Mathematical Problems in Engineering
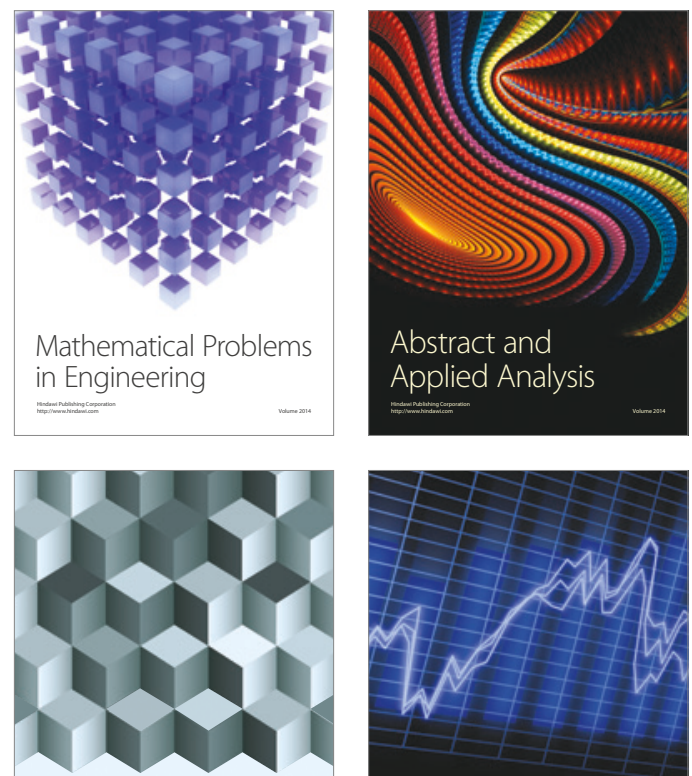

Journal of

Function Spaces

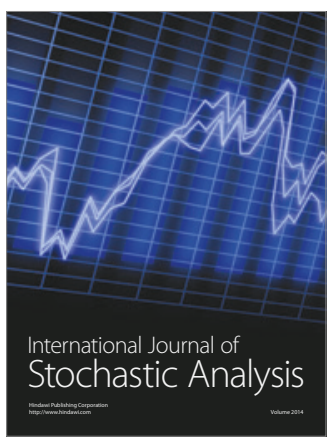

Probability and Statistics
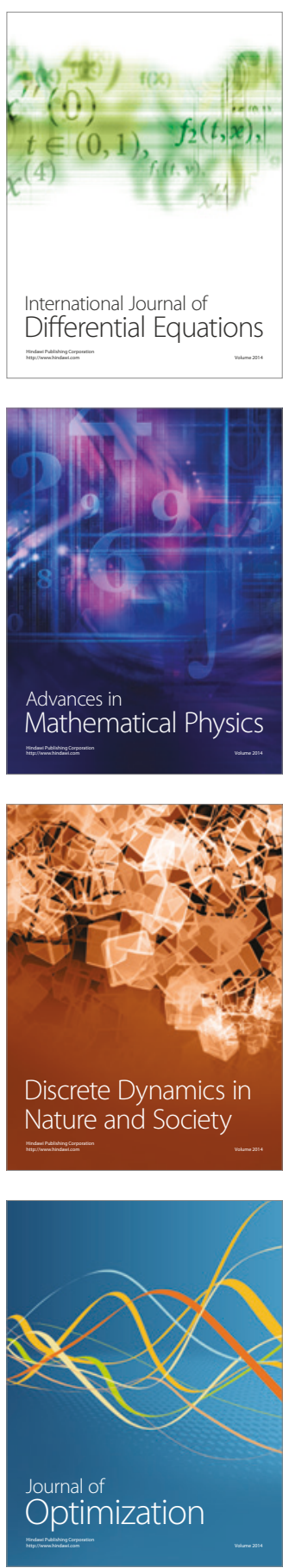